\title{
Epicardial Activation Increases Transmural Dispersion of Repolarization in a Heterogeneous Model of Wild-Type and Short QT Mutant Tissue
}

\author{
DL Weiss ${ }^{1}$, G Seemann ${ }^{1}$, FB Sachse ${ }^{2}$, O Doessel $^{1}$ \\ ${ }^{1}$ Institute of Biomedical Engineering, Universität Karlsruhe (TH), Karlsruhe, Germany \\ ${ }^{2}$ Nora Eccles Harrison Cardiovascular Research and Training Institute, University of Utah, USA
}

\begin{abstract}
This computational study investigates the effects of epicardial stimulation on healthy and mutant excitable tissue. A heterogeneous wild-type (WT) model of human ventricular electrophysiology and a modified version simulating short $Q T(S Q T)$ syndrome were used. The utilized anisotropic anatomical structures were both a slice cut out of a human left ventricular geometry and a beam from endocardium to epicardium extracted from this slice.

Epicardial pacing increased the transmural dispersion of repolarization (TDR) in WT and SQT mutant tissue. The QT time was prolonged in WT tissue but stayed nearly constant in SQT affected tissue. In the slice anatomy, intraventricular dispersion of repolarization overweights TDR. $T D R$ was increased nearby the electrode but reduced in tissue opposite to the electrode.

We assume, that during epicardial pacing the risk for arrhythmic events is increased but not higher in patients affected by the SQT syndrome compared to healthy patients.
\end{abstract}

\section{Introduction}

Epicardial activation of tissue as it is applied during biventricular pacing prolongs the QT interval and increases the transmural dispersion of repolarization (TDR) [1, 2]. This raised TDR augments the risk for the development of arrhythmic events. In 2004, a study investigating the genetic and biophysical basis of the short QT (SQT) syndrome was presented [3]. A N588K mutation in HERG protein was identified. This mutation increases the activity of the homogeneously distributed repolarizing $\mathrm{I}_{\mathrm{Kr}}$ current and therefore shortens action potentials (AP) and reduces TDR. Furthermore, ventricular fibrillation was easy to induce during electrophysiological studies in this tissue.

This computational study investigated, whether people affected by the SQT syndrome develop an increased risk for life threatening arrhythmias during epicardial pacing. Thus, the effect of epicardial pacing on wild-type tissue and tissue affected by the short QT syndrome was studied.

\section{Methods}

A computational model of a human ventricular myocyte forms the electrophysiological basis of this study [4]. This model describes ionic currents, ionic concentrations, and transmembrane voltage for one single cell by a set of coupled nonlinear ordinary differential equations. These equations were solved by a forward Euler method [5] with a time increment of $20 \mu \mathrm{s}$. The heterogeneous physiological (wild-type, WT) representation of cellular electrophysiology was created by modification of several ion channel properties in this model according to measurements on human cardiac myocytes [6]. The short QT 1 mutation was reproduced by inclusion of a new description for the rapidly activating delayed rectifier current $\mathrm{I}_{\mathrm{Kr}}$ within the WT model [7]. Due to the measurement data on cardiomyocytes affected by the SQT syndrome [8] a background substitution component replaced the voltagedependent inward-rectifying component in this representation. A faster activation and consequently an increased activity of $\mathrm{I}_{\mathrm{Kr}}$ was realized by a shift of the half-activation voltage of its activating rate constant to negative values and an increase of the amplyfing factor. A shift of the halfactivation voltage of the deactivating rate constant to positive values and an elevated intensifying factor led to an abolished rectification and an accelerated tail current decay of $\mathrm{I}_{\mathrm{Kr}}$ [7].

The single cell environment was transferred to a model of excitable tissue. Anisotropic electrical coupling between the cells was obtained by using the bidomain reaction-diffusion model [9]. The equations were solved by an iterative Gauss-Seidel method every $20 \mu$ s. Smooth transitions for the maximum conductances of the heterogeneously distributed ionic currents were included in the underlying model of cellular electrophysiology [6]. The virtual $\mathrm{M}$ cells were located near to the endocardium to obtain a realistic upright positive $\mathrm{T}$ wave in the transmurally computed ECG after endocardial stimulation [10].

The used anatomical structure was a three-dimensional slice of the Visible Female left ventricular data set. It was cut out perpendicular to the longitudinal axis of the ven- 


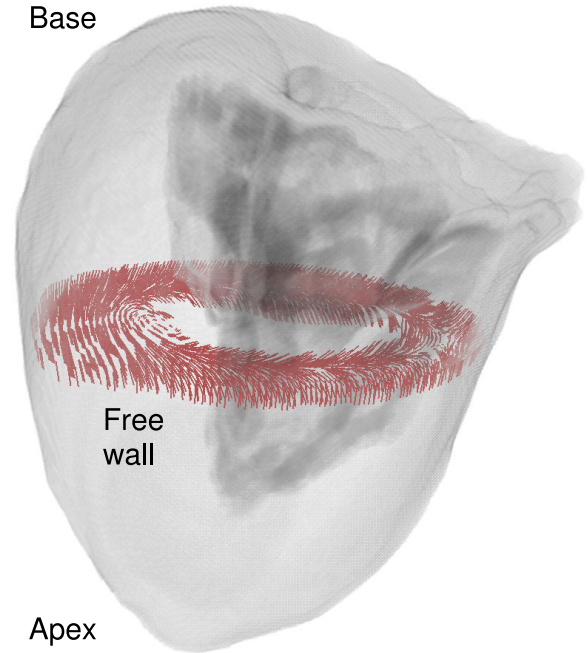

Figure 1. Human left ventricle obtained from the Visible Female data set. The highlighted cross-sectional slice perpendicular to the longitudinal axis was used to study the excitation conduction in a realistic anatomical structure under consideration of electrophysiological heterogeneity and electrical anisotropy. The rotating fiber orientation is depicted by lines in several exemplary voxels.

tricle and consisted of nearly 110.000 excitable cells surrounded by a bath. It is visualized in Fig. 1 within the whole left ventricle. Transmurally varying fiber orientation was reconstructed by means of a rule-based algorithm derived from anatomical studies [11]. Endocardial activation was initiated at transition points imitating the Purkinje fiber network. An epicardially attached electrode was simulated by applying currents in subepicardial myocytes nearby the electrode. The diameter of this electrode was set to $3 \mathrm{~mm}$. A long and thin line from endocardium to epicardium consisting of $96 \times 9 \times 9$ elements was extracted from this slice. It was used to investigate the principle impact of epicardial pacing on excitation conduction and repolarization in a less complex geometry. Both anatomical structures were composed of cubic voxels with an edge length of $0.2 \mathrm{~mm}$. Transmural ECGs were computed as the bath potential difference close to epicardial and endocardial border.

\section{Results}

Cells affected by the SQT syndrome elicited APs with markedly decreased duration due to a gain of function in the repolarizing $\mathrm{I}_{\mathrm{Kr}}$ current. APs in M cells were more shortened than in endocardial and epicardial cardiomyocytes leading to a reduced TDR [7].

Epicardial stimulation in general reversed the direction of the activation sequence and led to an inversion of the

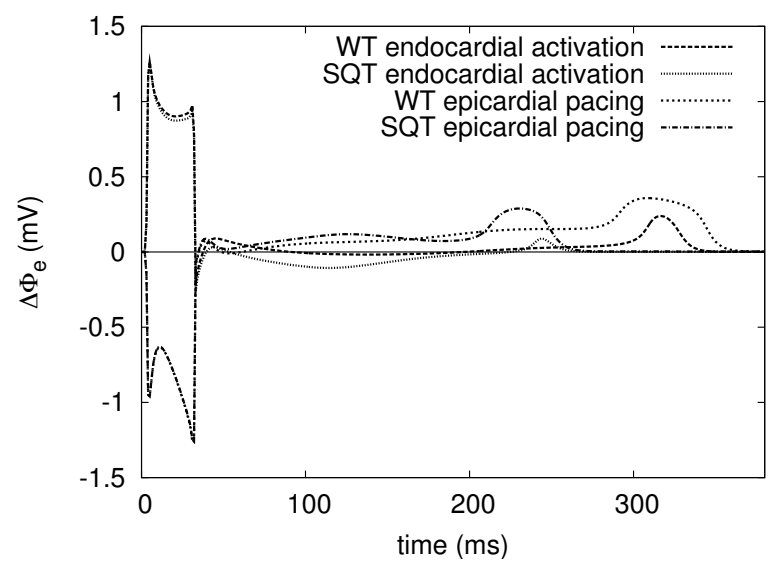

Figure 2. Transmural ECGs computed after endocardial activation and epicardial pacing in the simplified anatomical structure in WT and SQT mutant tissue. Epicardial stimulation emphasized the $\mathrm{T}$ wave indicating an increased TDR and prolonged the QT time in WT tissue.

QRS complex in the transmural ECG. This is shown for both WT and SQT mutant tissue in Figs. 2 and 3. The T wave was widened and its amplitude was augmented. This was due to the delayed activation and repolarization of the $\mathrm{M}$ cells and the earlier activation of the subepicardium. The temporal difference $t_{d}$ between the end and peak of the $\mathrm{T}$ wave as an index for the TDR [12] was increased in WT from 27 to $51 \mathrm{~ms}$ and in mutant tissue from 23 to 35 $\mathrm{ms}$ due to epicardial pacing. The time to full repolarization corresponding to the QT time was prolonged from 343 to $360 \mathrm{~ms}$ in WT tissue, but stayed nearly constant at $266 \mathrm{~ms}$ in SQT mutant tissue.

The activation sequence initiated by epicardially attached electrode started in the more realistic anatomy of the three-dimensional slice nearby the electrode and spread from there into the tissue (Fig. 5). The activation of adjacent myocytes was fastest in the midmyocardium of the slice because the helix angle of the fiber orientation is small in the midmyocardial region [11] and the increased excitation velocity along a muscle fiber is considered by the excitation conduction model. Thus, the depolarization of the tissue started in the midmyocardial region and conducted from there to the subendocardium and subepicardium in a certain distance from the pacing electrode (Fig. 5). This behavior was observed in both WT and SQT mutant tissue.

Transmural ECGs in the slice were simulated after epicardial pacing at two different positions. Lead P1 was computed as the extracellular potential difference nearby the epicardially attached pacing electrode, P2 described the transmural ECG computed on the opposing site of the electrode (Fig. 4). The QRS complexes were similar in 


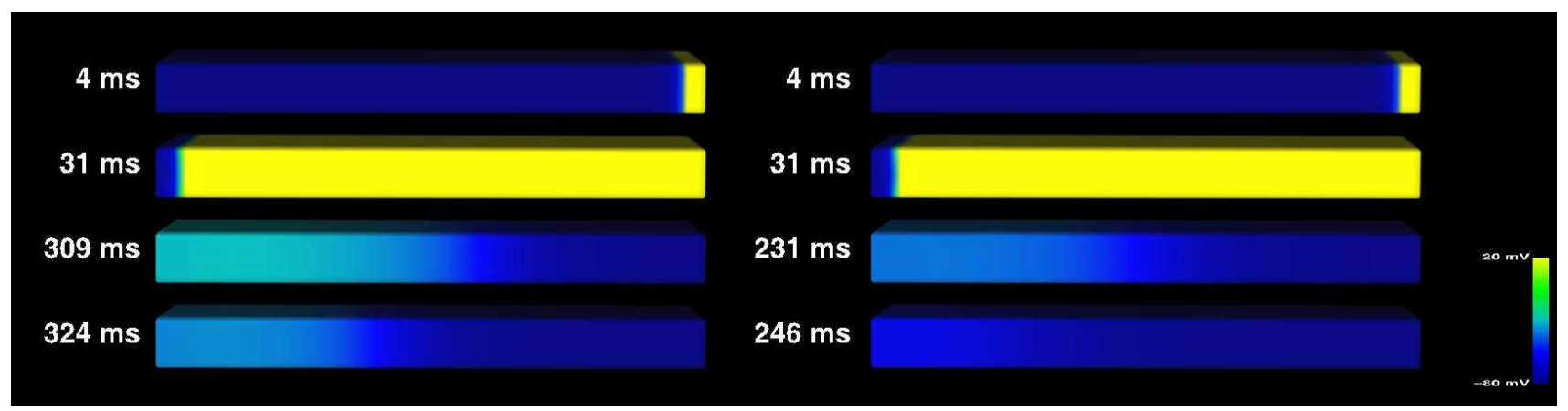

Figure 3. Transmembrane voltage distribution in a long and thin beam including heterogeneous electrophysiology from subendocardium (left side) to subepicardium (right side) at different time steps after the epicardial pacing impulse. The left column shows WT, the right column SQT mutant tissue. Bright color corresponds to activated tissue and dark depicts the resting potential of the cardiomyocytes. SQT mutation reduced the repolarization time. The last two rows show the distribution at the maximum of the $\mathrm{T}$ wave and $15 \mathrm{~ms}$ later. The more pronounced color gradient in WT tissue indicates the augmented TDR in this tissue compared to SQT mutant tissue.

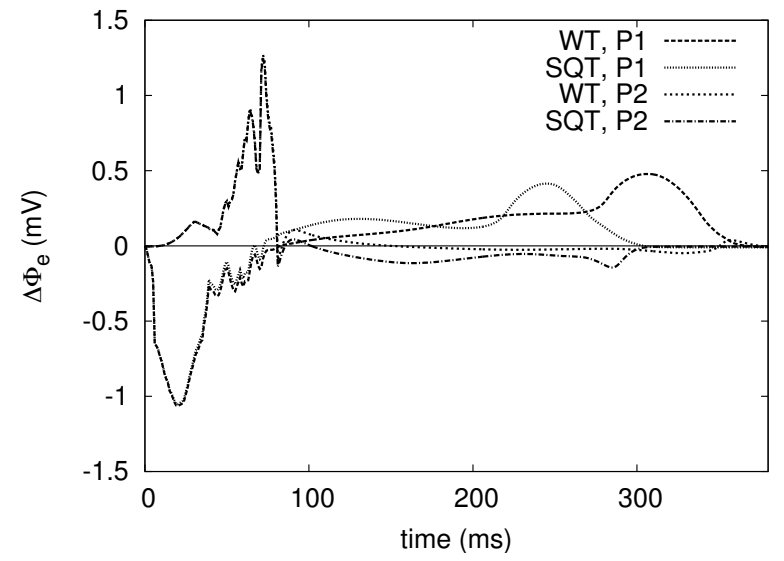

Figure 4. Transmural ECGs computed in the slice anatomy on two different positions after epicardial pacing in WT and SQT mutant tissue. P1 is the position nearby the pacing electrode, $\mathrm{P} 2$ is located on the opposing site.

both leads for both configurations of the cellular model indicating that the transmural activation sequence was not altered by the SQT mutation. In P1, the T wave was positive for both WT and SQT affected virtual tissue. Due to the reduced APD in cells affected by the SQT syndrome the onset of the $\mathrm{T}$ wave was earlier in mutant than in WT tissue. In P2, the QRS complexes were inverted revealing the altered transmural activation sequence. The onset of the QRS complexes in P2 was delayed for both representations of the electrophysiology due to the prolonged activation pathway. Due to the early activation of the M cells and the delayed stimulation of the surrounding tissue, the prolonged APD of the M cells was compensated leading to a homogenized repolarization. The $\mathrm{T}$ wave showed a small amplitude in WT and a higher amplitude but in- versed polarity in SQT mutant tissue. The SQT mutation slightly reduced $t_{d}$ from $50 \mathrm{~ms}$ in WT to $48 \mathrm{~ms}$ in position $\mathrm{P} 1$ but augmented $\mathrm{t}_{\mathrm{d}}$ from $4 \mathrm{~ms}$ to $12 \mathrm{~ms}$ in position $\mathrm{P} 2$.

\section{Discussion and conclusions}

Consistent with measurements [1,2], simulations in the simple nearly one-dimensional anatomy showed that epicardial pacing increased the TDR in WT as well as in SQT mutant tissue. The resulting TDR and therefore the risk for arrhythmogenic events due to epicardial pacing was reduced in SQT mutant compared to physiological tissue because the increase of TDR was more pronounced in WT tissue. The QT interval was prolonged in WT tissue due to epicardial pacing, while in SQT mutant tissue it was barely changed.

In the more complex ventricular slice, TDR after epicardial pacing mainly depended on the distance to the pacing electrode. It was augmented in WT and SQT case nearby the electrode. In the tissue opposite to the pacing site the altered transmural activation sequence clearly reduced the TDR due to the earlier activation of M cells in both configurations of the cellular model.

We assume that the substrate for development of a ventricular tachycardia provided by the intraventricular dispersion of repolarization due to altered activation pathway overweights the substrate provided by the TDR. If endocardial stimulation is not applicable, we expect that during epicardial pacing the risk for the development of Torsade de Pointes is increased but not higher in patients affected by the SQT syndrome compared to healthy patients. The effect of pacing position and frequency on the TDR has to be determined in future. 


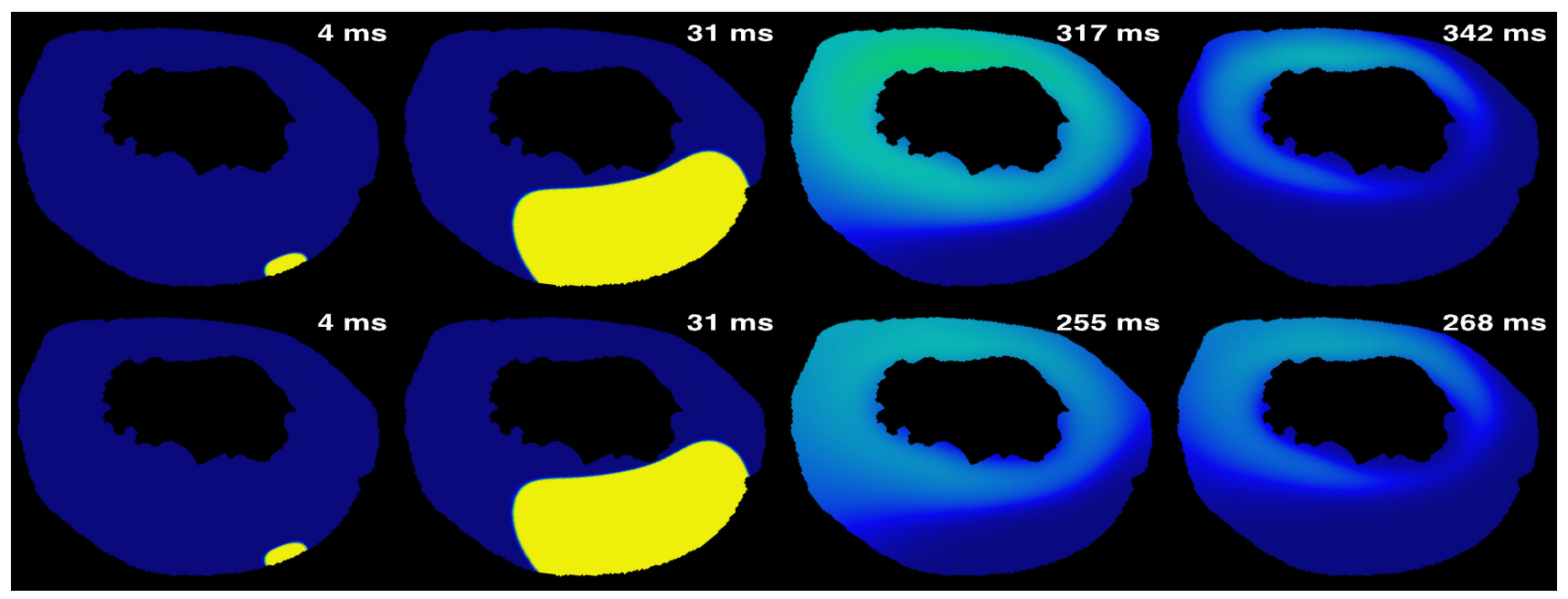

Figure 5. Excitation conduction and repolarization in the left ventricular slice obtained from the Visible Female data set at different time steps after epicardial pacing in WT (top) and SQT mutant tissue (bottom). The transmembrane voltage distribution is illustrated with the same color code as in Fig. 3. Epicardial pacing prolonged the activation pathway and thus increased the intraventricular dispersion of activation and repolarization. The TDR depended on the position within the tissue and its distance to the pacing electrode. Although the SQT mutation shortened the QT interval, the pattern of the repolarization is similar under both electrophysiological conditions.

\section{References}

[1] Fish JM, Di Diego JM, Nesterenko V, Antzelevitch C. Epicardial Activation of Left Ventricular Wall Prolongs QT Interval and Transmural Dispersion of Repolarization: Implications for Biventricular Pacing. Circ 2004;109(17):21362142.

[2] Medina-Ravell VA, Lankipalli RS, Yan GX, Antzelevitch C, Medina-Malpica NA, Medina-Malpica OA, Droogan C, Kowey PR. Effect of Epicardial or Biventricular Pacing to Prolong QT Interval and Increase Transmural Dispersion of Repolarization: Does Resynchronization Therapy Pose a Risk for Patients Predisposed to Long QT or Torsade de Pointes? Circ 2003;107(5):740-746.

[3] Brugada P, Brugada R, Antzelevitch C, Towbin JA, Brugada J. The brugada syndrome. In Zipes DP, Jalife J (eds.), Cardiac Electrophysiology. From Cell to Bedside, 4 edition. Philadelphia: W. B. Saunders Company, 2004; 625-632.

[4] Priebe L, Beuckelmann DJ. Simulation study of cellular electric properties in heart failure. Circ Res 1998;82:12061223.

[5] Press WH, Teukolsky SA, Vetterling WT, Flannery BP. Numerical Recipes in C. 2 edition. Cambridge, New York, Melbourne: Cambridge University Press, 1992.

[6] Seemann G, Sachse FB, Weiß DL, Dössel O. Quantitative reconstruction of cardiac electromechanics in human myocardium: Regional heterogeneity. J Cardiovasc Electrophysiol Oct. 2003;14(S10):S219-S228.

[7] Weiss DL, Seemann G, Sachse FB, Dössel O. Modelling of short QT syndrome in a heterogeneous model of the human ventricular wall. Europace 2005;7S2:105-117.

[8] Brugada R, Hong K, Dumaine R, Cordeiro J, Gaita F,
Borggrefe M, Menendez TM, Brugada J, Pollevick GD, Wolpert C, Burashnikov E, Matsuo K, Sheng Wu Y, Guerchicoff A, Bianchi F, Giustetto C, Schimpf R, Brugada P, Antzelevitch C. Sudden Death Associated With ShortQT Syndrome Linked to Mutations in HERG. Circ 2004; 109(1):30-35.

[9] Henriquez CS, Muzikant AL, Smoak CK. Anisotropy, fiber curvature and bath loading effects on activation in thin and thick cardiac tissue preparations: Simulations in a threedimensional bidomain model. J Cardiovasc Electrophysiol May 1996;7(5):424-444.

[10] Weiß DL, Seemann G, Dössel O. Conditions for equal polarity of $\mathrm{R}$ and $\mathrm{T}$ wave in heterogeneous human ventricular tissue. In Proc. BMT, volume 49-2/1. 2004; 364-365.

[11] Streeter DD. Gross morphology and fiber geometry of the heart. In Bethesda B (ed.), Handbook of Physiology: The Cardiovascular System, volume I. American Physiology Society, 1979; 61-112.

[12] Antzelevitch C. T peak-tend interval as an index of transmural dispersion of repolarization. Eur J Clin Invest Jul 2001;31(7):563-9.

Address for correspondence:

Dipl.-Ing. Daniel L. Weiss

Institute of Biomedical Engineering, Universität Karlsruhe (TH)

Kaiserstr. 12, 76128 Karlsruhe, Germany

tel./fax. ++49 721608 2790/2789

Daniel.Weiss@ibt.uni-karlsruhe.de 